\title{
Effects of ocean chemistry on chemical and morphological evolution of iron sulfide nanoparticles
}

\author{
SARICK MATZEN ${ }^{1}$, TUCKER ELY ${ }^{1}$, ADRIAN WACKET ${ }^{1}$, \\ AMY GARTMAN $^{2}$, CHRISTOPHER GERMAN ${ }^{3}$ AND \\ BRANDY M TONER ${ }^{1}$ \\ ${ }^{1}$ University of Minnesota \\ ${ }^{2}$ United States Geological Survey \\ ${ }^{3}$ Wood Hole Oceanographic Institution \\ Presenting Author: smatzen@umn.edu
}

Iron-containing nanoparticles emitted from deep sea hydrothermal vents are increasingly recognized as an important source of iron in modern oceans, and net hydrothermal contributions to the marine iron cycle are an active field of study in marine biogeochemistry. In particular, nanoparticles emanating from vents have large transport potentials and can record the (bio-)geochemical conditions at their point of formation, suggesting that iron-bearing particles may have untapped "biosignature" potential to act as tracers of lifeconducive conditions on extraterrestrial ocean worlds. Responsibly using particles as geochemical tracers is currently limited by our knowledge of the alteration and aggregation pathways that particles follow. For example, nanoparticle aggregation and the oxidation rates of iron containing complexes and minerals likely influence particle transport away from the vent source, as well as the bioavailability of exported iron. Iron speciation has been investigated previously in nanoparticles collected from hydrothermal vents, but the relationship between nanoparticle aggregation and oxidation can be better constrained to inform field interpretations and hydrothermal iron export models. Here we use synthesized pyrite nanoparticles and a highthroughput screening framework to investigate the effects of seawater composition, atmospheric oxidation state, and the presence/absence of organic ligands on pyrite nanoparticle aggregation and oxidation rates in the laboratory using a suite of analytical techniques (i.e. ferrozine method, laser particle size/dynamic light scattering analyses, and electron microscopy). All experiments were performed in parallel under conditions representative of Earth's modern ocean and more alkaline ocean worlds (e.g. Enceladus). The results help constrain the dynamic processes affecting iron export between 1-100 km away from hydrothermal vents on Earth, and may also inform future searches for particles that trace hydrothermal activity on icy ocean moons in the outer solar system. 